TRANSACTIONS OF THE

AMERICAN MATHEMATICAL SOCIETY

Volume 353, Number 2, Pages 441-456

S 0002-9947(00)02549-6

Article electronically published on August 3, 2000

\title{
HERMITIAN-EINSTEIN METRICS FOR VECTOR BUNDLES ON COMPLETE KÄHLER MANIFOLDS
}

\author{
LEI NI AND HUAIYU REN
}

\begin{abstract}
In this paper, we prove the existence of Hermitian-Einstein metrics for holomorphic vector bundles on a class of complete Kähler manifolds which include Hermitian symmetric spaces of noncompact type without Euclidean factor, strictly pseudoconvex domains with Bergman metrics and the universal cover of Gromov hyperbolic manifolds etc. We also solve the Dirichlet problem at infinity for the Hermitian-Einstein equations on holomorphic vector bundles over strictly pseudoconvex domains.
\end{abstract}

\section{INTRODUCTION}

The relation between the existence of Hermitian-Einstein metrics and stable vector bundles over compact Kähler manifolds is by now well understood due to work of Donaldson, Narasimhan-Seshadri, Simpson, Uhlenbeck-Yau and others (cf. [D1, [D3], [N-S], [Si], U-Y]). More recently, in [D2] the Dirichlet boundary value problem was solved for Hermitian-Einstein metrics, and many interesting applications were addressed. In this paper we study the existence of Hermitian-Einstein metrics for vector bundles over a class of complete Kähler manifolds. Roughly speaking we are able to show that if a vector bundle $E$ over a complete Kähler manifold $M$ admits an Hermitian metric which is asymptotically Hermitian-Einstein, then one can deform the metric to an Hermitian-Einstein metric, assuming that the spectrum of the Laplacian-Beltrami operator has positive lower bound (namely, $\lambda_{1}(M)>0$ ). More precisely, we can show that

Theorem 4.1. Let $M$ be a complete Kähler manifold, and let $\left(E, H_{0}\right)$ be an Hermitian vector bundle with Hermitian metric $H_{0}$. Assume that $\lambda_{1}(M)>0$, and that $\left|\Lambda F_{H_{0}}-\lambda I\right| \in L^{p}(M)$ for some real numbers $p>1$ and $\lambda$. Then there exists an Hermitian-Einstein metric $H$ on $E$ such that

$$
\Lambda F_{H}-\lambda I \equiv 0 .
$$

The above theorem can be thought as an analogue of the Cheng-Yau theorem on Kähler-Einstein metrics, since in [C-Y] they perturbed asymptotic Kähler-Einstein metrics into Kähler-Einstein metrics for strictly pseudoconvex domains. The examples satisfying the assumption of Theorem 4.1 include simply-connected strictly negative curved manifolds, Hermitian symmetric spaces of noncompact type and strictly pseudoconvex domains equipped with the Bergmann metric. According to Theorem 1.4.A of Gro the universal cover of any Kähler hyperbolic manifolds

Received by the editors November 5, 1998 and, in revised form, March 5, 1999.

2000 Mathematics Subject Classification. Primary 58G11.

Research was partially supported by an NSF grant. 
(in the sense of Gromov) satisfies the assumption of Theorem 4.1 too. Therefore Theorem 4.1 is applicable to a relatively broad class of Kähler manifolds. As an application we solve the Dirichlet problem at infinity for Hermitian-Einstein metrics for holomorphic vector bundles over strictly pseudoconvex domains. Similar theorems has been established previously for harmonic functions in [A-S by Anderson and Schoen, and for harmonic maps in [L-T by Li and Tam.

Theorem 4.4. Let $\Omega$ be a bounded strictly pseudoconvex domain in $\mathbf{C}^{\mathbf{n}}$, and let $\omega_{B}$ be the Bergmann metric on $\Omega$. Suppose that $E$ is a holomorphic vector bundle on $\bar{\Omega}$ and $H_{0}$ is a $C^{0}$-Hermitian metric of $E$ defined only on $\partial \Omega$. Then there exists a unique Hermitian-Einstein metric $H$ on $\Omega$ such that

$$
\left\{\begin{array}{l}
\Lambda F_{H} \equiv 0, \\
\lim _{x \rightarrow x_{0}} H(x)=H_{0}\left(x_{0}\right), \text { for any } x_{0} \in \partial \Omega .
\end{array}\right.
$$

We should point out that, using the approximation argument as in $\mathrm{A}-\mathrm{S}$, one can also solve the Dirichlet problem at infinity for Hermitian-Einstein metrics on vector bundles over simply-connected Kähler manifold $M$ with sectional curvature $-a^{2} \leq K_{M} \leq-b^{2}$. Therefore we can view our result as a nonlinear generalization of [A-S] to the system case. A similar theorem was proved before in [A-C-M] for harmonic maps.

When the manifold is a bounded strictly pseudoconvex domain in $\mathbf{C}^{\mathbf{n}}$ with complete Bergmann metric, analytically we have to deal with a degenerate semilinear system (a simple linear analogue will be the invariant Laplacian operator on unit balls). We solve this problem by the heat equation method. The heat flow method was also used by $\mathrm{Li}$ and Tam $[\mathrm{L}-\mathrm{T}]$ in proving the existence of harmonic maps between complete manifolds. However we take a different approach to establish the global existence for the heat equation. Instead of showing the local existence and then extending to a global solution, we use Donaldson's solution for the Dirichlet boundary value problem to establish global existence for heat equations of Hermitian-Einstein metrics. By the observation that the scaling argument of [D1] still works over compact domains we reduce the global existence of the heat equation to uniform $C^{0}$-estimates. Since $M$ is complete and even the scalar heat equation does not always have a long-time solution, we do need some assumptions on the initial data. This partially justifies our assumption in Theorem 4.1. In [D1] and [D2], since the manifold is compact the long-time existence of the heat-equation does not require any assumptions and is easier to find than in our case.

After we establish the global existence for the parabolic equation, the $L^{p}$ contraction of the scalar heat equation and the mean-value type inequalities of $\mathrm{Li}$ and Tam can be applied to prove the convergence of the solution of the heat equation to an Hermitian-Einstein metric.

The solution to the heat equation is in fact a family of Hermitian metrics on the holomorphic vector bundle under consideration. To prove the $C^{0}$-estimate we need a device to measure the "distance" between two metrics. In D1 Donaldson introduced a "distance" function to do this job. Although this "distance" function does not suit our needs, a modified version of it does, and that is the function $\rho(H, K)$ defined later in Section 3 (where $H$ and $K$ are two Hermitian metrics on the same vector bundle). However this $\rho(H, K)$ fails to serve as "distance" unless the determinant of $H K^{-1}$ equals 1 . In other words, the determinants of Hermitian metrics in the family obtained by solving the heat equation must be 
independent of the parameter $t$. This is in general not true unless the initial metric has vanishing scalar curvature. Fortunately we are able to prove that, as long as the scalar curvature of the initial Hermitian metric is an $L^{p}$ function for $p>1$ and $\lambda_{1}(M)>0$, one can always deform the metric conformally so that the resulting metric has vanishing scalar curvature. The main content of Section 2 is the proof of this fact. Section 3 consists of the proof of the global existence of the solution to the heat equation. In Section 4 we prove the convergence to an Hermitian-Einstein metric, Theorem 4.1 and Theorem 4.4.

Concerning our result, there are a few previous results which we should mention. First, in [Si] the existence of the Hermitian-Einstein metrics was proved for quasiprojective manifolds with incomplete Fubini-Study metrics under certain stability conditions. In $[\mathrm{B}$ an existence result was also proved for a so-called asymptotically flat manifold $M^{n}$ under the assumption that $\left|\Lambda F_{H_{0}}\right| \in L^{n}(M)$. Since our base manifolds are quite different from quasi-projective varieties or asymptotically flat manifolds, our theorem has no overlap with these results. Analytically, in [Si] the equations involved are nondegenerate and have a different nature from ours. On the other hand, after a slight modification on using the heat kernel our argument can be applied to give another proof of a more general version of the above-mentioned result in $[\mathrm{B}]$.

Acknowledgement. The authors would like to thank Professor Richard Wentworth for bringing this problem to their attention. The first author would like to express his gratitude to his thesis advisor, Professor Peter Li, for many valuable suggestions.

\section{Conformal Deformation}

Let $M^{n}$ be a Kähler manifold of complex dimension $n$, and let $(E, H)$ be a holomorphic vector bundle with an Hermitian metric $H$. Denote by $\omega=\sqrt{-1} g_{\alpha \bar{\beta}} d z^{\alpha} \wedge$ $d \bar{z}^{\beta}$ the Kähler form, where $g=g_{\alpha \bar{\beta}} d z^{\alpha} \otimes d \bar{z}^{\beta}$ is the Kähler metric. Then we can define the operator $\Lambda$ as the contraction with $-\sqrt{-1} \omega$, i.e., for any $(1,1)$-form $\sum a_{\alpha \bar{\beta}} d z^{\alpha} \wedge d \bar{z}^{\beta}$,

$$
\Lambda \sum a_{\alpha \bar{\beta}} d z^{\alpha} \wedge d \bar{z}^{\beta}=\sum g^{\alpha \bar{\gamma}} a_{\gamma \bar{\beta}} .
$$

A connection $A$ on the vector bundle $E$ is called Hermitian-Einstein (cf. [D1]) if the corresponding curvature form $F_{A}$ satisfies the following equation:

$$
\Lambda F_{A}=\lambda I,
$$

where $\lambda$ is some constant. In the case when $M$ is a compact Kähler manifold, $\lambda$ is a topological invariant (cf. [U-Y], [Siu ). If for an Hermitian metric $H$ on $E$ the corresponding Hermitian connection is Hermitian-Einstein, then the metric $H$ is called an Hermitian-Einstein metric (cf. [Siu]). Recall that under a holomorphic local frame $\left\{e_{i}\right\}$, if $H=\left(h_{i \bar{j}}\right)$, where $h_{i \bar{j}}=\left\langle e_{i}, e_{j}\right\rangle$, then the Hermitian connection $A_{H}$ and the corresponding curvature form $F_{H}$ are given by the following formulae:

$$
\begin{aligned}
A_{H} & =\partial H H^{-1}, \\
F_{H} & =\bar{\partial}\left(\partial H H^{-1}\right) .
\end{aligned}
$$

In this section we will show that by conformally deforming an initial metric $H_{0}$ on an Hermitian bundle $E$, i.e. by replacing $H_{0}$ with $e^{-\varphi} H_{0}$, we can obtain a new metric $H$ satisfying $\operatorname{tr}\left(\Lambda F_{H}\right)=0$. When $M$ is compact this follows trivially from the Hodge theory (cf. [Siu] $)$. We can show that under certain conditions this is also 
true for complete(noncompact) Kähler manifolds. This conformal deformation of the metric is our first step in proving the existence of Hermitian-Einstein metrics on complete Kähler manifolds.

Proposition 2.1. Let $M$ be a complete Kähler manifold of complex dimention n and let $\left(E, H_{0}\right)$ be a holomorphic vector bundle with Hermitian metric $H_{0}$. Assume the scalar curvature $S_{0}(x)=\operatorname{tr}\left(\Lambda F_{H_{0}}-\lambda I\right) \in L^{p}(M)$ for some $p>1$ and $\lambda$. Then there exists a function $\varphi$ such that the metric $H=e^{-\varphi} H_{0}$ satisfies

$$
\operatorname{tr}\left(\Lambda F_{H}-\lambda I\right) \equiv 0,
$$

provided that $\lambda_{1}(M)>0$, where $\lambda_{1}(M)$ is the lower bound of the first eigenvalue of the Laplacian-Beltrami operator.

Proof. Let $H=e^{-\varphi} H_{0}$. Direct calculation yields the following equalities:

$$
\begin{aligned}
A_{H} & =-e^{-\varphi} \partial \varphi H_{0} e^{\varphi} H_{0}^{-1}+e^{-\varphi} \partial H_{0} e^{\varphi} H_{0}^{-1}, \\
F_{H} & =-\bar{\partial} \partial \varphi I+F_{H_{0}} .
\end{aligned}
$$

Using the fact that $\Lambda \partial \bar{\partial} \varphi=\Delta \varphi$, we get

$$
\Lambda F_{H}=\Delta \varphi I+\Lambda F_{H_{0}} .
$$

Taking the trace on both sides, we get

$$
\operatorname{tr}\left(\Lambda F_{H}-\lambda I\right)=r \Delta \varphi+\operatorname{tr}\left(\Lambda F_{H_{0}}-\lambda I\right),
$$

where $r$ is the rank of the vector bundle $E$. Therefore it suffices to show that we can find $\varphi$ satisfying

$$
\Delta \varphi=-\frac{S_{0}(x)}{r} .
$$

By the method of solving the heat equation and using the heat-kernel estimate we know there exists such a $\varphi$ (cf. Theorem 2.7 of [N1] $)$. This proves the proposition.

\section{Global existence for the heat equation}

In the introduction we mentioned that the purpose of this paper is to prove the existence of a flat Hermitian-Einstein metric under certain growth condition. In other words we will solve the equation

$$
\Lambda F_{H}-\lambda I=0 .
$$

We use the heat equation method as Donaldson's approach in the compact case. In this section we show that there exists a global solution for the heat equation of (3.1).

In order to establish the global solution for the heat equation we use Donaldson's solution for the Dirichlet problem on compact domain and the compact exhaustion method. The key point here is to get a uniform estimate. In particular, we need to establish a uniform $C^{0}$-estimate. But before we go further we want to fix the notations and derive some formulae.

Let $M^{n}$ be a complete Kähler manifold of complex dimension $n$. Let $\left(E, H_{0}\right)$ be a holomorphic vector bundle on $M$ with Hermitian metric $H_{0}$. Consider a family of Hermitian metrics $H(t)$ on $E$ with the initial metric $H(0)=H_{0}$. Denote by $A_{H(t)}$ and $F_{H(t)}$ the corresponding connections and curvature forms. When there is no confusion, we will omit the parameter $t$ and simply write $H, A_{H}, F_{H}$ for $H(t), A_{H(t)}, F_{H(t)}$ respectively. We define a global section $h$ of the endomorphism 
bundle $\operatorname{End}(E)$ by $h=H H_{0}^{-1}$. Then $h_{0}=I$ and for sections $u, v$ of $E$ we have $\langle u, h v\rangle=\langle h u, v\rangle$. Using (2.2) and (2.3) we have the following formulae (cf. [Siu]):

$$
\begin{aligned}
A_{H}-A_{H_{0}} & =\partial h h^{-1}+h\left(\partial H_{0}\right) H_{0}^{-1} h^{-1}-\left(\partial H_{0}\right) H_{0}^{-1} \\
& =\left(\partial_{H_{0}} h\right) h^{-1} \\
F_{H}-F_{H_{0}} & =\bar{\partial}\left(\left(\partial_{H_{0}} h\right) h^{-1}\right),
\end{aligned}
$$

where $\partial_{H_{0}}$ is the $(1,0)$-component of the covariant derivative corresponding to the Hermitian connection for $H_{0}$.

The heat equation of (3.1) can be written in terms of $h$ as follows (cf. [Siu, D1]):

$$
\frac{\partial h}{\partial t} h^{-1}=-\Lambda F_{H}+\lambda I
$$

One can easily see that, written in local coordinates, this is a parabolic semilinear system. From [D2] we have the following existence theorem of Donaldson.

Theorem 3.1 (Donaldson). Let $E$ be a holomorphic vector bundle over a compact manifold $\Omega$ with non-empty boundary $\partial \Omega$. For any Hermitian metric $f$ on the restriction of $E$ to $\partial \Omega$ there is a unique metric $H$ on $E$ such that

(i) $H=f$ over $\partial \Omega$,

(ii) $\Lambda F_{H}=0$ in $\Omega$.

Remark. In fact it was proven in D2 that the heat equation (3.4) for the Dirichlet problem has a long time solution $H(t)$ for any initial metric $H_{0}$ such that $\left.H_{0}\right|_{\partial \Omega}=f$. Without any difficulty one can take the argument from [D2] to show that

Theorem 3.1'. Let $E$ be a holomorphic vector bundle over the compact manifold $\Omega$, with non-empty boundary $\partial \Omega$. For any Hermitian metric $f$ on the restriction of $E$ to $\partial \Omega$ there exists a solution metric $H(\cdot, t)$ on $E$ such that

$$
\left\{\begin{array}{l}
\frac{\partial h}{\partial t} h^{-1}=-\Lambda F_{H}+\lambda I \\
H(x, 0)=f(x) \\
\left.H(x, t)\right|_{\partial \Omega}=f(x) .
\end{array}\right.
$$

We will use this result in our proof of the existence for the heat equation. By the result of the last section we may assume that $\operatorname{tr} \Lambda F_{H_{0}} \equiv 0$. Then the next statement follows easily from the uniqueness of solutions for the scalar heat equation. One can consult [Siu] for a proof.

Lemma 3.2. Let $M$ be a complete Kähler manifold, and let $\Omega$ be a compact subdomain of $M$. Let $\left(E, H_{0}\right)$ be an Hermitian vector bundle with Hermitian metric $H_{0}$. Assume that $\operatorname{tr}\left(\Lambda F_{H_{0}}-\lambda I\right) \equiv 0$ for some real number $\lambda$ and $H$ is the solution of the heat equation (3.4) such that

(i) $H(x, 0)=H_{0}(x)$,

(ii) $\left.H(x, t)\right|_{\partial \Omega}=H_{0}(x)$.

Then $\operatorname{det}(h)(x)=1$, where $h=H H_{0}^{-1}$.

Using Lemma 3.2, we introduce a modified version of Donaldson's 'distance' on the space of Hermitian metrics as follows. 
First let $\tau(H, K)=\operatorname{tr}\left(H K^{-1}\right)$ as in [D1]. We define

$$
\rho(H, K)=\log \tau(H, K)+\log \tau(K, H)-2 \log r,
$$

where $r$ is the rank of $E$.

Lemma 3.3. If $H$ and $K$ are two Hermitian metrics on $E$ such that $\operatorname{det}\left(H K^{-1}\right) \equiv$ 1 , then $\rho(H, K) \geq 0$, and equality holds if and only if $H=K$.

Proof. Set $h=H K^{-1}$; then $h$ is a positive definite Hermitian matrix with $\operatorname{det} h=1$. Therefore we may assume $h$ is a diagonal matrix with positive eigenvalues $\lambda_{1}, \cdots, \lambda_{r}$ and $\lambda_{1} \cdots \lambda_{r}=1$. Now

$$
\begin{gathered}
\lambda_{1}+\cdots+\lambda_{r} \geq r \sqrt[r]{\lambda_{1} \cdots \lambda_{r}}=r, \\
\lambda_{1}^{-1}+\cdots+\lambda_{r}^{-1} \geq r \sqrt[r]{\lambda_{1}^{-1} \cdots \lambda_{r}^{-1}}=r,
\end{gathered}
$$

and equalities hold if and only if $\lambda_{1}=\cdots=\lambda_{r}=1$. Thus $\rho(H, K)=\log (\operatorname{tr} h)+$ $\log \left(\operatorname{tr} h^{-1}\right)-2 \log r \geq 0$, and equality holds only when $h=I$. This proves the lemma.

In order to prove the global existence of the heat equation we need to introduce more notations. Let $M$ and $\left(E, H_{0}\right)$ be as before. Let $\left\{\Omega_{i}\right\}_{i=1}^{\infty}$ be a exhausting sequence of compact subdomains of $M$, i.e. they satisfy $\Omega_{i} \subset \Omega_{i+1}$ and $\bigcup_{i}^{1} \Omega_{i}=M$. Using Donaldson's solutions of heat equations for the Dirichlet problem (see the remark following Theorem 3.1), we can find Hermitian metrics $H_{i}(x, t)$ on $\left.E\right|_{\Omega_{i}}$ for each $i$ such that

$$
\left\{\begin{array}{l}
\frac{\partial H_{i}}{\partial t} H_{i}^{-1}=-\Lambda F_{H_{i}}+\lambda I \\
H_{i}(x, 0)=H_{0}(x) \text { on } \Omega_{i} \\
\left.H_{i}(x, t)\right|_{\partial \Omega_{i}} \equiv H_{0}(x) \\
\lim _{t \rightarrow 1} \Lambda F_{H_{i}}-\lambda I=0
\end{array}\right.
$$

On each $\Omega_{i}$ we define $h_{i}=H_{i} H_{0}^{-1}$. We also define functions

$$
\rho_{i}(x, t)=\rho\left(H_{i}(t), H_{0}\right) \quad \text { and } \quad \rho_{i j}(x, t)=\rho\left(H_{i}(t), H_{j}(t)\right)
$$

for all $j>i$. Here $\rho$ is the 'distance' function defined by (3.5). We want to obtain a uniform bound for the functions $\rho_{i j}$ on compact subsets. Our approach is to establish $L^{p}$-estimates for $\rho_{i j}$ and then show that the $\rho_{i j}$ are in fact subsolutions of some heat equation. The $C^{0}$-estimates then follow from $L^{p}$ contraction of the heat equation and a mean-value type inequality. But first we need a few lemmas. The first lemma is the following differential inequality, which modifies an inequality in D1] or Siu. Since the proof is similar, we skip it here.

Lemma 3.4. On each $\Omega_{i}$ define $\hat{e}_{i}=\left|\Lambda F_{H_{i}}-\lambda I\right|^{2}$. Then we have

$$
\Delta \hat{e}_{i}-\frac{\partial \hat{e}_{i}}{\partial t} \geq 0
$$

In particular, $\sup _{\Omega_{i}} \hat{e}_{i}$ is a decreasing function in $t$.

The next lemma is analogous to inequality (1.9.2) in [Siu. One can prove it by using the argument in [Siu] with some minor changes. 
Lemma 3.5. Let $H_{i}$ and $h_{i}$ be as defined above. Then

$$
\Delta \log \operatorname{tr}\left(h_{i}\right) \geq-\left(\left|\Lambda F_{H_{0}}-\lambda I\right|+\left|\Lambda F_{H_{i}}-\lambda I\right|\right) .
$$

Lemma 3.6. Let $\rho_{i}(x, t), \rho_{i j}(x, t)$ be the functions defined as above. Assume that $\lambda_{1}(M)>0$ and $\left\|\Lambda F_{H_{0}}-\lambda I\right\|_{L^{p}(M)}<\infty$ for some $p>1$ and $\lambda$. Then for any fixed compact subset $K \subset \subset M$ there exists a constant

$$
C=C\left(K, \lambda_{1}(M), p,\left\|\Lambda F_{H_{0}}-\lambda I\right\|_{L^{p}(M)}\right)
$$

such that

$$
\left\|\rho_{i}(\cdot, t)\right\|_{L^{p}(K)} \leq C \quad \text { and } \quad\left\|\rho_{i j}(\cdot, t)\right\|_{L^{p}(K)} \leq C, \text { for any } j>i .
$$

Here $i$ is big enough so that $K \subset \Omega_{i}$.

Proof. We first show that $\rho_{i j}$ is bounded by $\rho_{i}$ and $\rho_{j}$. Recall that, by definition,

$$
\rho_{i j}(x, t)=\log \operatorname{tr}\left(H_{i} H_{j}^{-1}\right)+\log \operatorname{tr}\left(H_{j} H_{i}^{-1}\right)-2 \log r,
$$

but

$$
\begin{aligned}
\log \operatorname{tr}\left(H_{i} H_{j}^{-1}\right) & =\log \operatorname{tr}\left(H_{i} H_{0}^{-1} H_{0} H_{j}^{-1}\right) \\
& \leq \log \left(\operatorname{tr}\left(H_{i} H_{0}^{-1}\right) \operatorname{tr}\left(H_{0} H_{j}^{-1}\right)\right) \\
& =\log \operatorname{tr}\left(H_{i} H_{0}^{-1}\right)+\log \operatorname{tr}\left(H_{0} H_{j}^{-1}\right) .
\end{aligned}
$$

Similarly,

$$
\log \operatorname{tr}\left(H_{j} H_{i}^{-1}\right) \leq \log \operatorname{tr}\left(H_{j} H_{0}^{-1}\right)+\log \operatorname{tr}\left(H_{0} H_{i}^{-1}\right) .
$$

Thus by substituting these inequalities into the definition of $\rho_{i j}$ we have

$$
\rho_{i j}(x, t) \leq \rho_{i}(x, t)+\rho_{j}(x, t)+2 \log r .
$$

Next we show that $\log \operatorname{tr}\left(H_{0} H_{i}^{-1}\right)=\log \operatorname{tr}\left(h_{i}^{-1}\right)$ is bounded by $\log \operatorname{tr}\left(h_{i}\right)$. Again we assume that $h_{i}$ is diagonal with diagonal entries $\lambda_{1}, \cdots, \lambda_{r}$. Since $\lambda_{1} \cdots \lambda_{r}=1$, we have

Therefore

$$
\frac{1}{\lambda_{k}}=\lambda_{1} \cdots \lambda_{k-1} \lambda_{k+1} \cdots \lambda_{r} \leq\left(\operatorname{tr}\left(h_{i}\right)\right)^{r-1}
$$

and

$$
\operatorname{tr}\left(h_{i}^{-1}\right) \leq r\left(\operatorname{tr}\left(h_{i}\right)\right)^{r-1}
$$

$$
\log \operatorname{tr}\left(h_{i}^{-1}\right) \leq(r-1) \log \operatorname{tr}\left(h_{i}\right)+\log r .
$$

It remains to verify that $\log \operatorname{tr}\left(h_{i}\right) \in L^{p}\left(\Omega_{i}\right)$ and its norm is bounded uniformly on the fixed compact subset $K$. To do this we only need to estimate the function $\log \operatorname{tr}\left(h_{i}\right)-\log r$, since the $L^{p}$ norm of the constant $\log r$ does not affect the uniformity of our estimates. By Lemma 3.5 we know that

$$
\Delta \log \operatorname{tr}\left(h_{i}\right) \geq-\left(\left|\Lambda F_{H_{0}}-\lambda I\right|+\left|\Lambda F_{H_{i}}-\lambda I\right|\right),
$$

and also that $\log \operatorname{tr}\left(h_{i}\right)-\left.\log r\right|_{\partial \Omega_{i}}=0$ according to the definition of $h_{i}$. In order to simplify our notation we denote $\log \operatorname{tr}\left(h_{i}\right)-\log r$ simply by $g$. Multiplying by $g^{p-1}$ on both sides of the above differential inequality, we have

$$
(\Delta g) g^{p-1} \geq-\left(\left|\Lambda F_{H_{0}}-\lambda I\right|+\left|\Lambda F_{H_{i}}-\lambda I\right|\right) g^{p-1} .
$$

Integrating by parts, we get

$$
\int_{\Omega_{i}}\left(\left|\Lambda F_{H_{0}}-\lambda I\right|+\left|\Lambda F_{H_{i}}-\lambda I\right|\right) g^{p-1} \geq(p-1) \int_{\Omega_{i}}|\nabla g|^{2} g^{p-2} .
$$


On the other hand, by the assumption on the positivity of the first eigenvalue on $M$ we have

$$
\frac{p^{2}}{4} \int_{\Omega_{i}}|\nabla g|^{2} g^{p-2}=\int_{\Omega_{i}}\left|\nabla g^{\frac{p}{2}}\right|^{2} \geq \lambda_{1}(M) \int_{\Omega_{i}} g^{p} .
$$

Combining (3.7) and (3.8) and using the Hölder inequality, we then have

$$
\begin{aligned}
& \left(\int_{\Omega_{i}}\left(\left|\Lambda F_{H_{0}}-\lambda I\right|+\left|\Lambda F_{H_{i}}-\lambda I\right|\right)^{p}\right)^{\frac{1}{p}}\left(\int_{\Omega_{i}} g^{p}\right)^{\frac{p-1}{p}} \\
& \quad \geq \int_{\Omega_{i}}\left(\left|\Lambda F_{H_{0}}-\lambda I\right|+\left|\Lambda F_{H_{i}}-\lambda I\right|\right) g^{p-1} \\
& \quad \geq \frac{4(p-1) \lambda_{1}(M)}{p^{2}} \int_{\Omega_{i}} g^{p} .
\end{aligned}
$$

Therefore

$$
\|g\|_{L^{p}\left(\Omega_{i}\right)} \leq C(M, p)\left(\left\|\Lambda F_{H_{0}}-\lambda I\right\|_{L^{p}(M)}+\left\|\Lambda F_{H_{i}}-\lambda I\right\|_{L^{p}\left(\Omega_{i}\right)}\right) .
$$

By the $L^{p}$-contraction of the heat equation and Lemma 3.4 we have

$$
\left\|\Lambda F_{H_{i}}-\lambda I\right\|_{L^{p}\left(\Omega_{i}\right)} \leq\left\|\Lambda F_{H_{0}}-\lambda I\right\|_{L^{p}(M)} .
$$

Here we have used the fact that $\left|\Lambda F_{H_{i}}-\lambda I\right|^{2}$ is a subsolution of the heat equation, due to Lemma 3.4, with Dirichlet boundary data at $\partial \Omega_{i}$, due to the assumption that $H$ satisfies (3.6). This proves our lemma.

Remark. From Lemma 3.6 we have that on any compact subset $K \subset M$ the norms $\left\|\rho_{i j}\right\|_{L^{2 p}(K)}$ are uniformly bounded from above by some constant independent of $j$ and $t$.

Before we prove the main theorem of this section we show that $\rho_{i j}$ is a subsolution of the heat equation.

Proposition 3.7. For all $j>i$, the functions $\rho_{i j}(x, t)$ satisfy the following differential inequalities on $\Omega_{i}$ :

$$
\left(\Delta-\frac{\partial}{\partial t}\right) \rho_{i j}(x, t) \geq 0 .
$$

Proof. Let $H(t), K(t)$ be two families of Hermitian metrics on $E$ satisfying the same conditions as do $H_{i}, H_{j}$. Set $\tau=\operatorname{tr}\left(H K^{-1}\right)$ and $u=\log \tau$. If we show that inequality (3.9) holds with $\rho_{i j}$ replaced by $u$, the proposition will follow immediately. Now

$$
\Delta u-\frac{\partial}{\partial t} u=\frac{\Delta \tau}{\tau}-\frac{\tau_{t}}{\tau}-\frac{|\nabla \tau|^{2}}{\tau^{2}},
$$

where $\tau_{t}=\partial \tau / \partial t$. Since $\tau=\operatorname{tr}\left(H K^{-1}\right)$, we have

$$
\begin{aligned}
\tau_{t} & =\operatorname{tr}\left(H_{t} K^{-1}-H K^{-1} K_{t} K^{-1}\right) \\
& =\operatorname{tr}\left(H_{t} H^{-1} H K^{-1}-H K^{-1} K_{t} K^{-1}\right) \\
& =\operatorname{tr}\left(-\Lambda F_{H} H K^{-1}+H K^{-1} \Lambda F_{K}\right) \\
& =\operatorname{tr}\left(\Lambda\left(F_{K}-F_{H}\right) H K^{-1}\right),
\end{aligned}
$$

where we used the fact that equation (3.4) can also be written as $H_{t} H^{-1}=-\Lambda F_{H}$, and that $\operatorname{tr}(A B)=\operatorname{tr}(B A)$. 
To simplify notations we set $h=H K^{-1}$ for the rest of the proof. Using formula (3.3) with $H_{0}$ replaced by $K$, we obtain

$$
\begin{aligned}
\left(F_{H}-F_{K}\right) H K^{-1} & =\bar{\partial}\left(\partial_{K} h h^{-1}\right) h \\
& =\bar{\partial} \partial_{K} h+\partial_{K} h h^{-1} \bar{\partial} h .
\end{aligned}
$$

Hence

$$
\tau_{t}=\Delta \operatorname{tr}(h)-\operatorname{tr}\left(\Lambda\left(\partial_{K} h h^{-1} \bar{\partial} h\right)\right)
$$

or

$$
\Delta \tau-\frac{\partial \tau}{\partial t}=\operatorname{tr}\left(\Lambda\left(\partial_{K} h h^{-1} \bar{\partial} h\right)\right)
$$

Substituting this back into (3.10), we have

$$
\Delta u-\frac{\partial u}{\partial t}=\frac{\operatorname{tr}\left(\Lambda\left(\partial_{K} h h^{-1} \bar{\partial} h\right)\right)}{\tau}-\frac{|\nabla \tau|^{2}}{\tau^{2}} .
$$

Now the only thing we need to verify is

$$
\tau \operatorname{tr}\left(\Lambda\left(\partial_{K} h h^{-1} \bar{\partial} h\right)\right) \geq|\nabla \tau|^{2} .
$$

This can be checked by direct calculation in local coordinates, and is done on page 25 of Siu].

Now we can state and prove the main theorem of this section.

Theorem 3.8. Let $M$ be a complete Kähler manifold, and let $\left(E, H_{0}\right)$ be an Hermitian vector bundle with metric $H_{0}$. Then the heat equation (3.4) has a global solution if $\lambda_{1}(M)>0$ and $\left\|\Lambda F_{H_{0}}-\lambda I\right\|_{L^{p}(M)}<\infty$ for some $p>1$ and $\lambda \in \mathbf{R}$.

Proof. First we use the $L^{p}$-estimates of Proposition 3.7 and Lemma 3.6 to derive a $C^{0}$-estimate for $\rho_{i j}$. The interested reader may consult [N2] for a detailed proof. We only sketch the proof here.

Let $K$ be a compact subset of $M$ and $T>0$. We want to show that there is a positive constant $C=C(M, T, K)$ such that

$$
\sup _{K \times[0, T]} \rho_{i j} \leq C(T, M, p, i) \text {, for any } j>i \text { uniformly. }
$$

This can be done as follows.

Without loss of generality we can assume that $K=B_{o}(r)$. For $j>i>>1$ we can assume that $B_{o}(4 r) \subset \Omega_{i}$. By Proposition 3.7 we know that $\rho_{i j}(x, t)$ are subsolutions of the scalar heat equations. Now we can extend the $\rho_{i j}(x, t)$ to $t<0$ simply by defining them to be zero, using the fact that

$$
\rho_{i j}(x, 0) \equiv 0
$$

Then we get $\rho_{i j}(x, t)$ defined on $B_{o}(2 r) \times(-1,1)$ and subsolutions of the scalar heat equation. Now we can apply the mean-value inequality of Li and Tam for the subsolutions of the heat equation. When necessary we can do a translation along the time direction to apply Theorem 4.1 of $[\mathrm{L}-\mathrm{T}]$. After applying the mean-value inequality we have

$$
\sup _{B_{o}(r) \times[0, T]} \rho_{i j}^{p}(x, t) \leq C(M, r, T) \sup _{0 \leq t \leq T} \int_{B_{o}(4 r)} \rho_{i j}^{p}(y, t) d y .
$$

Combining this with Lemma 3.6, we have the estimates (3.11). 
Next we apply Donaldson's scaling argument in Lemma 19 of [D1] to obtain the $C^{1}$-estimate on $K \times\left[T_{0}, T\right]$, where $T_{0}>0$. More precisely, we show that there exists a constant $C=C_{1}\left(M, T, T_{0}, K\right)$ such that

$$
\sup _{K \times\left[T_{0}, T\right]}\left|\nabla H_{i}\right|(x, t) \leq C\left(M, T, T_{0}, K\right) .
$$

In order to apply Lemma 19 of [D1] we need to verify that $\sup _{K \times\left[T_{0}, T\right]}\left|\Lambda F_{H_{j}}-\lambda I\right|$ is bounded from above uniformly and $H_{j}$ converges uniformly after passing to subsequence. Since $\left|\Lambda F_{H_{j}}-\lambda I\right|^{2}$ is a subsolution of the scalar heat equation, the uniform boundness basically follows from the same argument as the one for $\rho_{i j}$. The only difference here is that instead of Theorem 1.1 we apply Theorem 1.2 of $[\mathrm{L}-\mathrm{T}$, a mean-value type inequality with initial data involved. We leave the details to the interested reader. Now we show that, after choosing a subsequence, the $H_{j}$ converge uniformly. This can be done as follows. First let $h^{(j)}=H_{j} H_{i}^{-1}$ and $\tau=\operatorname{tr}\left(h^{(j)}\right)$. Direct calculation as before shows that over $B_{o}(r) \times\left[T_{0}, T\right]$, we have

$$
\begin{aligned}
\Delta \tau= & \operatorname{tr}\left(\left(\Lambda F_{H_{i}}-\lambda I\right) h^{(j)}\right)+\operatorname{tr}\left(\left(\Lambda F_{H_{j}}-\lambda I\right) h^{(j)}\right) \\
& +\operatorname{tr}\left(\Lambda\left(\partial_{H_{i}} h^{(j)}\right)\left(h^{(j)}\right)^{-1}\left(\bar{\partial} h^{(j)}\right)\right) \\
\geq & -C_{1}\left(\left|\Lambda F_{H_{i}}-\lambda I\right|+\left|\Lambda F_{H_{j}}-\lambda I\right|\right)+C_{2} \operatorname{tr}\left(\Lambda\left(\partial_{H_{i}} h^{(j)}\right)\left(\bar{\partial} h^{(j)}\right)\right) .
\end{aligned}
$$

Here we have used the estimate (3.11) and the fact that $\operatorname{det}\left(h^{(j)}\right) \equiv 1$, which together with (3.11) implies that $\left(h^{(j)}\right)^{-1} \geq C_{2} I$. In the above inequality the $C_{i}$ are constants derived from (3.11) and independent of $j$. Now let

$$
e\left(h^{(j)}\right)=\operatorname{tr}\left(\Lambda\left(\partial_{H_{i}} h^{(j)}\right)\left(\bar{\partial} h^{(j)}\right)\right) .
$$

Using the above inequality and the fact that $\sup _{K \times\left[T_{0}, T\right]}\left|\Lambda F_{H}-l I\right|$ is bounded from above uniformly, we can write

$$
\Delta \tau \geq-C_{1} C_{3}+C_{2} e\left(h^{(j)}\right)
$$

on $B_{o}(r) \times\left[T_{0}, T\right]$. Now multiply the last inequality by $\tau \varphi^{2}$ and integrate it over $M$, where $\varphi$ is a cut-off function which equals 1 in $B_{o}(r / 2)$ and is supported in $B_{o}(r)$. We then obtain

$$
\int_{M}(\Delta \tau) \tau \varphi^{2} \geq-C_{1} C_{3} \int_{M} \tau \varphi^{2}+C_{2} \int_{M} e\left(h^{(j)}\right) \tau \varphi^{2} .
$$

Integrating by parts, using the Schwarz inequality, and then integrating along the time direction, we have

$$
\int_{T_{0}}^{T} \int_{M}|\nabla \varphi|^{2} \tau^{2}+C_{1} C_{3} \int_{T_{0}}^{T} \int_{M} \tau \varphi^{2} \geq \int_{T_{0}}^{T} \int_{M} e\left(h^{(j)}\right) \tau \varphi^{2} .
$$

Now we can use the estimate (3.11) again and the fact that $\operatorname{det}\left(h^{(j)}\right) \equiv 1$, which implies that $\tau \geq r$, to get the following estimate:

$$
\int_{T_{0}}^{T} \int_{B_{o}(r / 2)} e\left(h^{(j)}\right) \leq C_{4} .
$$

Because the $h^{(j)}$ are Hermitian and have uniform $C^{0}$ bound, (3.13) will imply that the $h^{(j)}$ are uniformly bounded in $L_{1}^{2}\left(B_{o}(r / 2) \times\left[T_{0}, T\right]\right)$. Using the fact that 
$L_{1}^{2}\left(B_{o}(r / 2) \times\left[T_{0}, T\right]\right)$ is compact in $L^{2}\left(B_{o}(r / 2) \times\left[T_{0}, T\right]\right)$, by passing to a subsequence, we have that the $H_{j}$ converge in $L^{2}\left(\left(B_{o}(r / 2) \times\left[T_{0}, T\right]\right)\right.$. Consequently,

$$
\int_{T_{0}}^{T} \int_{B_{o}(r / 2)} \rho_{j k}^{2} \leq \epsilon
$$

for $j, k$ sufficiently large. Applying the mean-value inequality to $\rho_{j k}^{2}$, we have (notice that $\rho_{j k}$ is a subsolution of the heat equation)

$$
\sup _{B_{o}(r / 4) \times\left[T_{0}, T\right]} \rho_{j k} \leq C_{5} \epsilon,
$$

which implies that $H_{j}$ converges uniformly to an Hermitian metric $H_{\infty}$. Since we have verified all the necessary conditions of Lemma 19 of [D1], the scaling argument there proves (3.12). Now that we have the estimates (3.11) and (3.12), the standard elliptic (or parabolic) theory (cf. G-T]) is enough to establish our theorem. This is similar to the case for the harmonic map equation. The linear theory we use here is the Schauder theory coupled with an $L^{p}$ theory.

\section{Existence of the Hermitian-Einstein metrics.}

In this section we prove the existence of the Hermitian-Einstein metrics. Since we have established the global existence of the heat equation, we only need to show that the solution to the heat equation (3.4) converges to a solution to the elliptic equation (3.1).

Theorem 4.1. Let $M$ be a complete Kähler manifold, and $\left(E, H_{0}\right)$ be an Hermitian vector bundle with Hermitian metric $H_{0}$. Assume that $\lambda_{1}(M)>0$, and that $\left\|\Lambda F_{H_{0}}-\lambda I\right\|_{L^{p}(M)}<\infty$ for some $p>1$ and $\lambda$. Then there exists an HermitianEinstein metric $H$ on $E$ such that

$$
\Lambda F_{H} \equiv \lambda I .
$$

Proof. By Theorem 3.8 we know that there exists an Hermitian metric $H(t)$ on $E$ such that $h=H H_{0}^{-1}$ satisfies

$$
\frac{\partial h}{\partial t} h^{-1}=-\Lambda F_{H}+\lambda I .
$$

To prove our theorem we only need to show that $\hat{e}(x, t)=\left|\Lambda F_{H}-\lambda I\right|^{2} \rightarrow 0$ as $t \rightarrow \infty$. In fact we can show that $\hat{e}(x, t)$ decays exponentially, thanks to the heat kernal estimate of Li and Tam.

Proof of exponential decay. First, as shown in [L-T], we have the following estimate for the heat kernel $K(x, y, t)$ :

$$
\int_{M} K^{q}(x, y, t) d y \leq C_{2} \exp \left(-\frac{4 \lambda_{1}(M)(q-1)}{q}(t-1)\right) .
$$

This was shown in [L-T] under a few technical assumptions on the manifold $M$. But in fact it is still true if we merely assume the positivity of $\lambda_{1}(M)$. For example, one can consult [Gri] for a proof and a state of the art survey on heat kernel estimates.

On the other hand, if we squeeze a little harder than [D1] and [Siu], we can show that $\hat{e}^{\frac{1}{2}}(x, t)$ satisfies

$$
\left(\Delta-\frac{\partial}{\partial t}\right) \hat{e}^{\frac{1}{2}}(x, t) \geq 0 .
$$

Note that this is a sharper version of Lemma 3.4. The proof is as follows. 
By the calculation on page 31 of [Siu], we have

$$
\left(\Delta-\frac{\partial}{\partial t}\right) \hat{e}(x, t) \geq 2\left|\nabla\left(\Lambda F_{H}\right)\right|^{2} .
$$

To prove the above sharper inequality we only need to verify that

$$
\left|\nabla\left(\Lambda F_{H}\right)\right|^{2} \geq|\nabla| \Lambda F_{H}||^{2}
$$

which can be checked by direct calculation.

Now we can show that $\hat{e}^{\frac{1}{2}}(x, t)$ decays exponentially:

$$
\begin{aligned}
\hat{e}^{\frac{1}{2}}(x, t) & \leq \int_{M} K(x, y, t) \hat{e}^{\frac{1}{2}}(y, 0) d y \\
& \leq\left(\int_{M} K^{q}(x, y, t) d y\right)^{\frac{1}{q}}\left(\int_{M} \hat{e}^{\frac{p}{2}}(y, 0) d y\right)^{\frac{1}{p}} \\
& =\left(\int_{M} K^{q}(x, y, t) d y\right)^{\frac{1}{q}}\left(\int_{M}\left|\Lambda F_{H_{0}}\right|^{p}(y, 0) d y\right)^{\frac{1}{p}} .
\end{aligned}
$$

Combining this with (4.1), we have proved that $\hat{e}(x, t)$ decays exponentially. Thus we complete the proof of the theorem.

Remark. One can easily show that the Hermitian-Einstein metric we constructed in Theorem 4.1 is a bounded $L^{p}$-distance away from the initial metric $H_{0}$. Also among the class of metrics which are a bounded $L^{p}$ distance away from $H_{0}$ it can be shown that there exists at most one Hermitian-Einstein metric. It was pointed out to us by H. Donnelly that one can show Theorem 4.1 directly, using the elliptic version of our argument in the proof of Theorem 3.8. On the other hand, we believe that the existence of the solution to the Hermitian-Einstein heat equation should hold under more relaxed assumptions than that of Theorem 3.8. For example, it should be true for $\left\|\Lambda F_{H_{0}}-\lambda I\right\|_{\infty} \leq 1$. One can also obtain the similar theorems when $\left|\Lambda F_{H_{0}}-\lambda I\right|$ satisfies the assumption as in Theorem 2.7 of [N1] and $M$ has nonnegative Ricci curvature with suitable volume growth, as in [N1. In other words, we believe that the nonlinear Hermitian-Einstein equation is solvable once the corresponding linear equation for the determinant line bundle is solvable. The linear equation has been solved in [N1] for various cases (cf. Theorems 2.7, 2.8, 2.9 of [N1]).

When $M$ is of complex dimension 2 the above theorem can be rephrased as an existence result for the anti-self-dual connection. In Guo, the Yang-Mills fields equation was studied for holomorphic bundles over cylindrical four manifolds. Our corollary gives an existence result for a class of complex surfaces which are different from the cylindrical ones.

Corollary 4.2. Let $M$ be a complete Kähler surface and let $\left(E, H_{0}\right)$ be a holomorphic vector bundle with Hermitian metric $H_{0}$. Assume that $\lambda_{1}(M)>0$ and $\left|\Lambda F_{H_{0}}\right| \in L^{p}(M)$ for some $p>1$. Then there exists an anti-self-dual $U(r)$ connection on $E$, where $r$ is the rank of $E$.

Proof. The proof is a direct consequence of Theorem 4.1. We only need to observe that an Hermitian connection is anti-self-dual if the corresponding curvature form $F$ is of type $(1,1)$ and belongs to the kernel of $\Lambda$, which is satisfied by the Hermitian connection we constructed in Theorem 4.1 . 
The same calculation as in the compact case gives the following inequalities of the Chern numbers:

Corollary 4.3. Let $M$ and $E$ be the Kähler manifold and vector bundle in Theorem 4.1. If $H$ is the Hermitian-Einstein metric constructed in Theorem 4.1, then

$$
\int_{M}\left(c_{2}(E, H)-\frac{r-1}{2 r} c_{1}(E, H)^{2}\right) \wedge \phi^{n-2} \geq 0 .
$$

In the following we will use Theorem 4.1 to solve the Dirichlet problem at infinity for the Hermitian-Einstein equation. More precisely we are going to prove the following theorem;

Theorem 4.4. Let $\Omega$ be a bounded strictly pseudoconvex domain in $\mathbf{C}^{\mathbf{n}}$, and let $\emptyset_{B}$ be the Bergmann metric on $\Omega$. Suppose that $E$ is a holomorphic vector bundle on $\bar{\Omega}$ and $H_{0}$ is a $C^{0}$-Hermitian metric of $E$ defined only on $\partial \Omega$. Then there exists a unique Hermitian-Einstein metric $H$ on $\Omega$ such that

$$
\left\{\begin{array}{l}
\Lambda F_{H} \equiv 0, \\
\lim _{x \rightarrow x_{0}} H(x)=H_{0}\left(x_{0}\right), \text { for any } x_{0} \in \partial \Omega .
\end{array}\right.
$$

The proof of the theorem will be a direct application of Theorem 4.1 if we can establish the fact that $\lambda_{1}\left(\Omega, \emptyset_{B}\right)>0$ for the strictly pseudoconvex domain $\Omega$ with the Bergmann metric $\omega_{B}$. But this positivity of the lower bound for the spectrum of the Laplacian operator was verified for a class of Kähler metrics in [Do. In fact, using an observation of Gromov [Gro, it was essentially proved in Do that the Laplacian operator $\Delta: L_{2} \Lambda^{i}(\Omega) \rightarrow L_{2} \Lambda^{i}(\Omega)$ has positive lower bound on its spectrum for $i \neq n$ (cf. [Do, Proposition 4.4]), where $n$ is the complex dimension of $\Omega$. Here, for the sake of the completeness, we include a simple proof for the Laplacian operator on functions.

Proposition 4.5. Let $\Omega$ be a bounded strictly pseudoconvex domain in $\mathbf{C}^{\mathbf{n}}$. If $\varphi$ is the plurisubharmonic defining function of $\Omega$, then for the complete Kähler metric defined by $g_{\alpha \bar{\beta}}=\partial^{2} g / \partial z^{\alpha} \partial \bar{z}^{\beta}$, where $g=-\log (-\varphi)$, we have $\lambda_{1}(\Omega, g) \geq n^{2} / 4$. Consequently, $\lambda_{1}\left(\Omega, \omega_{B}\right)>0$ for the Bergmann metric $\omega_{B}$, since the Bergmann metric is quasi-isometric to $\mathrm{g}$.

Proof. First we can trivially write

$$
\Delta g=n .
$$

Therefore, for any subdomain $\Sigma \subset \subset \Omega$ we have

$$
\begin{aligned}
n \operatorname{Vol}(\Sigma) & =\int_{\Sigma} \Delta g d v=\int_{\partial \Sigma} \frac{\partial g}{\partial \nu} d A \\
& \leq \int_{\partial \Sigma}|\nabla g| d A \leq A(\partial \Sigma) .
\end{aligned}
$$

The last inequality follows from the fact that $|\nabla g|^{2} \leq 1$, which can be verified by direct calculation. One can also consult [C-Y] for details. By Cheeger's inequality (cf. $[\underline{\mathrm{S}-\mathrm{Y}}]$ ) we can conclude that

$$
\lambda_{1}(\Omega, g) \geq \frac{n^{2}}{4} .
$$


Proof of Theorem 4.4. It will be sufficient to prove the theorem for the $C^{2}$ metric first. The general case follows from an approximation argument of $[\mathrm{A}-\mathrm{S}$, the maximum principle and an ellpitic version of our $C^{0}$ estimates in the last section. Therefore let us assume that $H_{0}$ is a $C^{2}$ metric of $E$ on the boundary. First we extend $H_{0}$ to $\Omega$ smoothly to get a $C^{2}$ Hermitian metric on $\left.E\right|_{\bar{\Omega}}$. We still denote this metric by $H_{0}$. Now we need to verify that $\left|\Lambda F_{H_{0}}\right| \in L^{p}(M)$ for some $p>1$. Since the metric $g$ in Proposition 4.5 is quasi-isometric to the Bergmann metric, we only need to check it using the metric $g$. Direct calculation shows that

$$
\begin{aligned}
\left|\Lambda F_{H_{0}}\right|^{2} & =\sum_{i j}\left(\sum_{\alpha \beta} g^{\alpha \bar{\beta}}\left(F_{H_{0}}\right)_{i, \alpha \bar{\beta}}^{j}\right)^{2} \\
& \sim O\left(\varphi^{2}\right) .
\end{aligned}
$$

Therefore we have $\left|\Lambda F_{H_{0}}\right| \in L^{p}(M)$ for $p>n$.

Now we can apply Theorem 4.1 to conclude that we can deform $H_{0}$ into an Hermitian-Einstein metric $H_{\infty}(x)=H(x, \infty)$, where $H(x, t)$ is the solution of the corresponding heat equation. The only thing left to show is that $H_{\infty}(x)$ and $H_{0}(x)$ share the same boundary data. This can be done by showing that

$$
\lim _{x \rightarrow \partial \Omega} \rho\left(H_{\infty}, H_{0}\right)=0 .
$$

By definition we have

$$
\begin{aligned}
\rho\left(H(x, t), H_{0}(x)\right) & =\log \frac{\operatorname{tr}\left(H H_{0}^{-1}\right) \operatorname{tr}\left(H_{0} H^{-1}\right)}{r^{2}} \\
& =\log \frac{\operatorname{tr}(h) \operatorname{tr}\left(h^{-1}\right)}{r^{2}},
\end{aligned}
$$

where $h=H H_{0}^{-1}$ satisfies

$$
\frac{\partial h}{\partial t} h^{-1}=-\Lambda F_{H} .
$$

Direct calculation shows that

$$
\frac{\partial}{\partial t} \log \operatorname{tr}(h) \leq\left|\Lambda F_{H}\right| .
$$

Similarly, using the equation

$$
\frac{\partial h^{-1}}{\partial t} h=\Lambda F_{H},
$$

we get

$$
\frac{\partial}{\partial t} \log \operatorname{tr}\left(h^{-1}\right) \leq\left|\Lambda F_{H}\right| .
$$

Hence

$$
\begin{aligned}
\rho\left(H(x, T), H_{0}(x)\right) & =\log \operatorname{tr}(h)+\log \left(\operatorname{tr}\left(h^{-1}\right)\right)-2 \log r \\
& =\int_{0}^{T} \frac{\partial}{\partial t} \log \operatorname{tr}(h) d t+\int_{0}^{T} \frac{\partial}{\partial t} \log \operatorname{tr}\left(h^{-1}\right) d t \\
& \leq 2 \int_{0}^{T}\left|\Lambda F_{H}\right| d t .
\end{aligned}
$$


Then

$$
\begin{aligned}
\rho\left(H_{1}, H_{0}\right) & \leq 2 \int_{0}^{1}\left|\Lambda F_{H}\right| d t \\
& =2\left(\int_{0}^{T}+\int_{T}^{\infty}\right)\left|\Lambda F_{H}\right| d t .
\end{aligned}
$$

Since $\left|\Lambda F_{H}\right|$ is a subsolution of the heat equation with $L^{p}$ initial value, the mean value inequality of $\mathrm{Li}$ and Tam (cf. $[\mathrm{L}-\mathrm{T}$, Theorem 1.2]) shows that

$$
\lim _{x \rightarrow \partial \Omega} \sup _{[0, T]}\left|\Lambda F_{H}\right|(x, t)=0 .
$$

Here we also used the fact that $\lim _{x \rightarrow \partial \Omega}\left|\Lambda F_{H_{0}}\right|=0$, which follows from (4.3). On the other hand, from the proof of Theorem 4.1, we know that $\left|\Lambda F_{H}\right|$ decays exponentially. Therefore, for any $\epsilon>0$, we can find a $T$ such that

$$
\int_{T}^{\infty}\left|\Lambda F_{H}\right| d t \leq \epsilon
$$

Combining the above two facts, we have the proof of the existence part of our theorem. The uniqueness follows easily from the subharmonicity of $\rho\left(H_{1}, H_{2}\right)$ and the maximum principle, if $H_{1}$ and $H_{2}$ are two solutions of (4.2).

Remark. As final remarks, we should point out that Theorem 4.4 remains true if one replaces the Bergmann metrics by complete Kähler-Einstein metrics, and Theorem 4.1 together with Theorem 4.4 can be generalized to Yang-Mills-Higgs equations, thanks to the Bochner type inequalities in [Si]. The approximation argument of A-S can be applied here to establish a similar theorem for the existence of the Dirichlet problem at infinity for the case when $M$ is a simply-connected Kähler manifold with sectional curvature $-a^{2} \leq K_{M} \leq-b^{2}$. We just state the theorem as follows, and leave the detailed proof to the interested reader.

Theorem 4.6. Let $M$ be a complete simply-connected Kähler manifolds with sectional curvature $-a^{2} \leq K_{M} \leq-b^{2}<0$, and $\partial M$ the ideal boundary at infinity. Suppose that $E$ is an Hermitian vector bundle defined on $\bar{M}=M \cup \partial M$ and $H_{0}$ is a $C^{0}$-Hermitian metric of $E$ defined only on $\partial M$. Then there exists a unique Hermitian-Einstein metric $H$ on $M$ such that

$$
\left\{\begin{array}{l}
\Lambda F_{H} \equiv 0, \\
\lim _{x \rightarrow x_{0}} H(x)=H_{0}\left(x_{0}\right), \text { for any } x_{0} \in \partial M .
\end{array}\right.
$$

\section{REFERENCES}

[A-S] M. Anderson \& R. Schoen, Positive harmonic functions on complete manifolds of negative curvature, Annals of Math. 121(1985), 429-461. MR 87a:58151

[A-C-M] P. Avilés, H. I. Choi and M. Micallef, Boundary behavior of harmonic maps on nonsmooth domains and complete negatively curved manifolds, J. Funct. Anal. 99(1991), 293-331. MR 92j:58025

[B] S. Bando, Einstein-Hermitian metrics on noncompact Kähler manifolds, Einstein metrics and Yang-Mills connections (Sanda, 1990), 27-33, Lecture Notes in Pure and Appl. Math. Dekker, New York, 1993. MR 94d:32040

[B-S] S. Bando \& S. T. Siu, Stable sheaves and Einstein-Hermitian metrics, Geometry and analysis on complex manifolds, 39-50, World. Sci. Publishing, River Edge, NY, 1994. MR 98e:32053 
[C-Y] S. Y. Cheng \& S. T. Yau, On the existence of a complete Kähler manifolds and the regularity of Fefferman's equation, Commun. Pure Appl. Math. 33(1980), 507-544. MR 82f:53074

[D1] S. K. Donaldson, Anti Self-Dual Yang-Mills Connections over Complex Algebraic Surfaces and Stable Vector Bundle, Proc. London Math. Soc. (3), 50(1985), 1-26. MR 86h:58038

[D2] S. K. Donaldson, Boundary value problems for Yang-Mills fields, Journal of Geometry and Physics, 8(1992), 89-122. MR 93d:53033

[D3] S. K. Donaldson, Infinite determinants, stable bundles and curvature, Duke Math. J.54(1987), 231-147. MR 88g:32046

[Do] H. Donnelly, $L_{2}$ cohomology of pseudoconvex domains with complete Kähler metrics, Michigan Math. J. 41(1994), 433-442. MR 95h:32007

[G-H] P. Griffiths \& J. Harris, Principles of Algebraic Geometry, John Wiley, New York, 1978. MR 80b:14001

[G-T] D. Gilbarg \& N. S. Trudinger, Elliptic partial differential equations of second order, 2nd edition, Springer-Verlag, 1983. MR 86c:35035

[Gri] A. Grigor'yan, Heat kernel upper bounds on a complete noncompact manifold, Rev. Math. Iberoamericana. 10(1994), 395-452 MR 96b:58017

[Gro] M. Gromov, Kähler hyperbolicity and $L_{2}$-Hodge theory, J. Diff. Geom. 33(1991), 263292. MR 92a:58133

[Guo] G-Y Guo, Yang-Mills fields on cylindrical manifolds and holomorphic bundles I, Commun. Math. Phys. 179(1996), 737-775. MR 98a:32040

$[\mathrm{K}] \quad$ S. Kobayashi, Differential Geometry of Complex Vector Bundles, Princeton, 1986. MR 89e:53100

[L1] P. Li, Lecture notes on geometric analysis, Research Institute of Mathematics, Global Analysis Center Seoul National University, 1993. MR 96m:58269

[L-T] P. Li \& L. F. Tam, The heat equation and harmonic maps of complete manifolds, Invent. Math. 105(1991), 1-46. MR 93e:58039

[N1] L. Ni, Vanishing theorems on complete Kähler manifolds and their applications, J. Diff. Geom. 50(1998), 89-122. CMP 99:10

[N2] L. Ni, Hermitian harmonic maps from complete Hermitian manifolds to complete Riemannian manifolds, Math. Z. 232(1999), 331-355. CMP 2000:04

[N-S] M. S. Narasimhan \& C. S. Seshadri, Stable and unitray vector bundles on a compact Riemann surface, Annals of Math. 82(1965), 540-564. MR 32:1725

[Si] C. T. Simpson, Constructing variations of Hodge structure using Yang-Mills theory and applications to uniformization, JAMS. 1 (1988), 867-918. MR 90e:58026

[Siu] Y. T. Siu, Lectures on Hermitian-Einstein metrics for stable bundles and Kähler-Einstein metrics, Birkhäuser, Basel-Boston, 1987. MR 89d:32020

[S-Y] R. Schoen \& S. T. Yau, Lectures on Differential Geometry, Academia Sinica Press, Beijing, 1988; English transl., Internat. Press, Cambridge, MA, 1994. MR 97d:53001

[U-Y] K. Uhlenbeck \& S. T. Yau, On the existence of Hermitian-Yang-Mills connections in stable vector bundles, Comm. Pure Appl. Math. 39(1986), 257-293. MR 88c:58154

Department of Mathematics, Purdue University, West Lafayette, Indiana 47907

E-mail address: Ini@math.purdue.edu

Current address: Department of Mathematics, Stanford University, Stanford, California 94305

Department of Mathematics, University of California, Irvine, California 92697

E-mail address: hren@math.uci.edu 